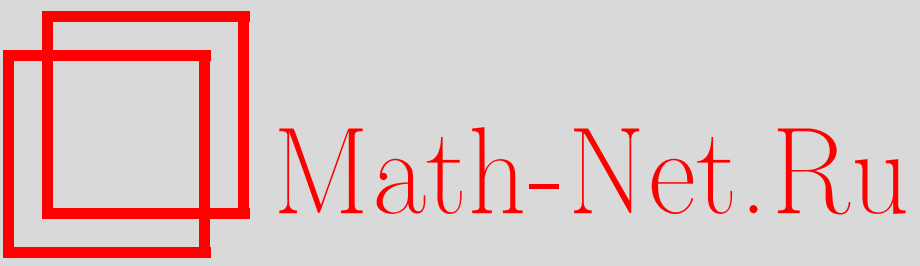

Г. Трибель, Пространства на множествах, УМH, 2005, том 60, выпуск 6, 187-206

DOI: https://doi.org/10.4213/rm1683

Использование Общероссийского математического портала Math-Net.Ru подразумевает, что вы прочитали и согласны с пользовательским соглашением

http: //www.mathnet.ru/rus/agreement

Параметры загрузки:

IP : 18.209 .158 .208

26 апреля 2023 г., 17:54:52 


\title{
ПРОСТРАНСТВА НА МНОЖЕСТВАХ
}

\author{
Г. ТРИБЕЛЬ
}

В работе рассматриваются пространства $\mathfrak{B}_{p q}^{s}$ и $\mathfrak{F}_{p q}^{s}$ положительной гладкости $s>0$, основанные на $L_{p}$-пространствах с $0<p \leqslant \infty$ и воспроизводящих формулах для гладких функций. Мы сравниваем эти пространства с $B$-пространствами и $F$-пространствами, полученными другими средствами.

Библиография: 37 названий.

\section{СОДЕРЖАНИЕ}

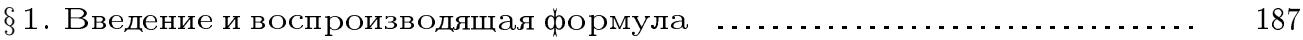

$\S 2$. Пространства, определяемые с помощью анализа Фурье . . . . . . . . . 189

2.1. Основные обозначения …................................... 189

2.2. Кварковые разложения и фреймы всплесков .................... 190

§3. Доказательство теоремы 1, дальнейшие комментарии . ............. 192

3.1. Доказательство теоремы 1 . .................................. 192

3.2. Свойство мултиразрешения …............................... 194

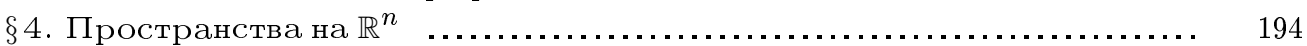

4.1. Определение и основные утверждения … . . . . . . . . . . . . . . . . . . 194

4.2. Свойства . ................................................. 197

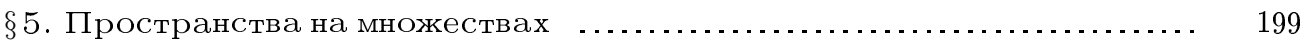

5.1. Предварительные сведения, пространства последовательностей ...... 199

5.2. Функциональные пространства .............................. 202

5.3. Свойства, комментарии, преобразования типа "снежинки" ........... 203

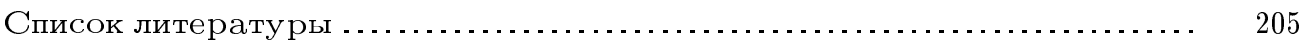

\section{§1. Введение и воспроизводящая формула}

Теория классических пространств Никольского-Бесова $B_{p q}^{s}$, пространств Соболева $W_{p}^{s}$ и пространств бесселевых потенщиалов $H_{p}^{s}$ положительной гладкости $s>0$, в том виде, в котором она развита в [24], [23], [3], основана на теории $L_{p}$-пространств с $1 \leqslant p \leqslant \infty,($ дробных ) производных и разностях функций. В этой статье мы описываем другой подход к пространствам типа $B_{p q}^{s}$ и $F_{p q}^{s}$ (также положительной гладкости $s>0)$, основанный на теории $L_{p}$-пространств с $0<p \leqslant \infty$ и воспроизводящих формулах для гладких функций. Этот метод примени́м к $\mathbb{R}^{n}$, к областям в $\mathbb{R}^{n}$, замкнутым множествам в $\mathbb{R}^{n}$ и абстрактным квазиметрическим пространствам. Мы сравниваем результат с другими подходами. 
Пусть $k$ - неотрицательная бесконечно дифференцируемая функция на $\mathbb{R}^{n}$ с носителем

$$
\operatorname{supp} k \subset\left\{y \in \mathbb{R}^{n}:|y|<2^{J-\varepsilon}, y>0\right\}
$$

при некотором $\varepsilon>0$ и $J \in \mathbb{N}$ (можно фиксировать значение $J=n$ ), и пусть

$$
\sum_{m \in \mathbb{Z}^{n}} k(x-m)=1, \quad x \in \mathbb{R}^{n} .
$$

Тогда

$$
k^{\beta}(x)=\left(2^{-J} x\right)^{\beta} k(x) \geqslant 0, \text { если } x \in \mathbb{R}^{n} \text { и } \beta \in \mathbb{N}_{0}^{n} .
$$

Пусть

$$
k_{j m}^{\beta}(x)=k^{\beta}\left(2^{j} x-m\right), \quad \text { где } j \in \mathbb{N}_{0} \quad \text { и } m \in \mathbb{Z}^{n} .
$$

Пусть $K$ - другая функция того же типа, удовлетворяющая условиям (1) и (2), и пусть $K^{\beta}$ и $K_{j m}^{\beta}$ - такие же, как в (3) и (4). Пусть $(f, g)=f(g)$, где $f \in S^{\prime}\left(\mathbb{R}^{n}\right)$ и $g \in S\left(\mathbb{R}^{n}\right)$.

Tеорема 1. Существуют такие универсальнье функиии $\Psi_{j, r ; m, l}^{\beta, \gamma} \in S\left(\mathbb{R}^{n}\right)$ c $\beta \in \mathbb{N}_{0}^{n}, \gamma \in \mathbb{N}_{0}^{n} ; j \in \mathbb{N}_{0}, r \in \mathbb{N}_{0} ; m \in \mathbb{Z}^{n}, l \in \mathbb{Z}^{n}$, ито для любой введенной више пары функиий $k, K$ вьполняется соотношение

$$
K_{r l}^{\gamma}(x)=\sum_{\beta, j \geqslant r, m}\left(K_{r l}^{\gamma}, \Psi_{j, r ; m, l}^{\beta, \gamma}\right) k_{j m}^{\beta}(x), \quad x \in \mathbb{R}^{n},
$$

где ряд сходится безусловно в $S\left(\mathbb{R}^{n}\right)$. Для любьх $\varkappa>0, M>0, b>0$ существует такая положительная постоянная $c=c_{\varkappa, M, b}$ (зависящая от $\left.k u K\right)$, что

$$
\left|\left(K_{r l}^{\gamma}, \Psi_{j, r ; m, l}^{\beta, \gamma}\right)\right| \leqslant c 2^{-\varepsilon|\gamma|} 2^{-\varkappa|\beta|} 2^{-(j-r) M}\left(1+\left|m-2^{j-r} l\right|\right)^{-b} .
$$

Это выражение одной системы $\left\{K_{r l}^{\gamma}\right\}$ через другую систему $\left\{k_{j m}^{\beta}\right\}$ с помошью комплексных коэффициентов, удовлетворяющих условию (6), дает возможность ввести пространства

$$
\mathfrak{B}_{p q}^{s}\left(\mathbb{R}^{n}\right) \quad \text { и } \mathfrak{F}_{p q}^{s}\left(\mathbb{R}^{n}\right) \quad \text { для } s>0, \quad 0<p \leqslant \infty, \quad 0<q \leqslant \infty
$$

(с $p<\infty$ для $\mathfrak{F}$-пространств) как подпространства в $L_{p}\left(\mathbb{R}^{n}\right)$ с помощью представлений

$$
f=\sum_{\beta, j, m} \lambda_{j m}^{\beta} k_{j m}^{\beta}
$$

где $\left\{\lambda_{j m}^{\beta}\right\} \subset \mathbb{C}$ принадлежит некоторому пространству последовательностей. Это делается в $\S 4$. Но сначала, в $\S 2$, мы приводим сведения о (сейчас почти классических) пространствах $B_{p q}^{s}\left(\mathbb{R}^{n}\right)$ и $F_{p q}^{s}\left(\mathbb{R}^{n}\right)$, определяемых с помошью анализа Фурье. На основе этих результатов мы доказываем теорему 1 в $\S 3$. Затем показьвается, что при $0<p \leqslant \infty$ (при $p<\infty$ для $F$-пространств) и при $0<q \leqslant \infty$ имеем

$$
\mathfrak{B}_{p q}^{s}\left(\mathbb{R}^{n}\right)=B_{p q}^{s}\left(\mathbb{R}^{n}\right) \text { при } s>\sigma_{p}=n\left(\frac{1}{p}-1\right)_{+}
$$

и

$$
\mathfrak{F}_{p q}^{s}\left(\mathbb{R}^{n}\right)=F_{p q}^{s}\left(\mathbb{R}^{n}\right) \text { при } s>\sigma_{p q}\left(\frac{1}{\min (p, q)}-1\right)_{+},
$$


в то время как пространства $\mathfrak{B}_{p q}^{s}\left(\mathbb{R}^{n}\right)$ и $\mathfrak{F}_{p q}^{s}\left(\mathbb{R}^{n}\right)$ при $p<1$ и $0<s<\sigma_{p}$ (как подпространства $L_{p}\left(\mathbb{R}^{n}\right)$ ), с одной стороны, и $B_{p q}^{s}\left(\mathbb{R}^{n}\right)$ и $F_{p q}^{s}\left(\mathbb{R}^{n}\right)$ (как подпространства $S^{\prime}\left(\mathbb{R}^{n}\right)$ (содержашие сингулярные распределения)), с другой стороны, не могут совпадать. Наконец, в $\S 5$ мы имеем дело с пространствами типа (7) на множествах в $\mathbb{R}^{n}$, главным образом на областях (= открытых множествах) $\Omega$ и на компактных множествах $\Gamma$, которые являются носителями конечных радоновских мер на $\mathbb{R}^{n}$. Мы снова сравниваем результат с пространствами, введенными другими средствами, и формулируем некоторые задачи, которые следует обсудить в дальнейшем.

\section{§. Пространства, определяемые с помощью анализа Фурье}

2.1. Основные обозначения. Мы используем стандартные обозначения: $\mathbb{R}^{n}$ (евклидово $n$-мерное пространство), $\mathbb{R}=\mathbb{R}^{1}, \mathbb{C}$ (комплексная плоскость), $\mathbb{Z}^{n}$ (решетка всех точек в $\mathbb{R}^{n}$ с целочисленными компонентами), $\mathbb{N}$ (натуральные числа), $\mathbb{N}_{0}$ (неотрицательные целые), $\mathbb{N}_{0}^{n}$ (мультииндексы), $x^{\beta}=x_{1}^{\beta_{1}} \cdots x_{n}^{\beta_{n}}$ (мономы с $x \in \mathbb{R}^{n}$ и $\left.\beta \in \mathbb{N}_{0}^{n}\right), S\left(\mathbb{R}^{n}\right)$ (пространство Шварца), $S^{\prime}\left(\mathbb{R}^{n}\right)$ (умеренные распределения), $\widehat{f}=F f$ (преобразование Фурье функции $\left.f \in S^{\prime}\left(\mathbb{R}^{n}\right)\right), f^{\vee}=F^{-1} f$ (соответствующее обратное преобразование Фурье). Пусть

$$
\left\|f \mid L_{p}\left(\mathbb{R}^{n}\right)\right\|=\left(\int_{\mathbb{R}^{n}}|f(x)|^{p} d x\right)^{1 / p}, \quad 0<p \leqslant \infty
$$

с очевидными изменениями для $p=\infty$. Мы предполагаем, что читатель знаком с основньми утверждениями о пространствах $B_{p q}^{s}\left(\mathbb{R}^{n}\right)$ и $F_{p q}^{s}\left(\mathbb{R}^{n}\right)$. Все необходимые (и более широкие) сведения можно найти в [27], [28], [35].

Пусть $\varphi_{0} \in S\left(\mathbb{R}^{n}\right)$, где $\varphi_{0}(x)=1$ при $|x| \leqslant 1$ и $\varphi_{0}(y)=0$ при $|y| \geqslant 3 / 2$. Пусть

$$
\varphi_{k}(x)=\varphi_{0}\left(2^{-k} x\right)-\varphi_{0}\left(2^{-k+1} x\right), \quad x \in \mathbb{R}^{n}, \quad k \in \mathbb{N} .
$$

Тогда $\sum_{j=0}^{\infty} \varphi_{j}(x)=1$ - диадическое разложение единицы и $\left(\varphi_{j} \widehat{f}\right)^{\vee}(x)$ - целая аналитическая функция для любой функции $f \in S^{\prime}\left(\mathbb{R}^{n}\right)$.

ОПРЕДЕЛЕНИЕ 2. Пусть последовательность $\varphi=\left\{\varphi_{j}\right\}_{j=0}^{\infty}$ такая же, как и выше. Пусть $0<p \leqslant \infty$ (с $p<\infty$ для $F$-пространств $), 0<q \leqslant \infty, s \in \mathbb{R}$. Тогда $B_{p q}^{s}\left(\mathbb{R}^{n}\right)-$ семейство всех функций $f \in S^{\prime}\left(\mathbb{R}^{n}\right)$, удовлетворяюших условию

$$
\left\|f \mid B_{p q}^{s}\left(\mathbb{R}^{n}\right)\right\|_{\varphi}=\left(\sum_{j=0}^{\infty} 2^{j s q}\left\|\left(\varphi_{j} \widehat{f}\right)^{\vee} \mid L_{p}\left(\mathbb{R}^{n}\right)\right\|^{q}\right)^{1 / q}<\infty
$$

и $F_{p q}^{s}\left(\mathbb{R}^{n}\right)$ - семейство всех функций $f \in S^{\prime}\left(\mathbb{R}^{n}\right)$, удовлетворяющих условию

$$
\left\|f\left|F_{p q}^{s}\left(\mathbb{R}^{n}\right)\left\|_{\varphi}=\right\|\left(\sum_{j=0}^{\infty} 2^{j s q}\left|\left(\varphi_{j} \widehat{f}\right)^{\vee}(\cdot)\right|^{q}\right)^{1 / q}\right| L_{p}\left(\mathbb{R}^{n}\right)\right\|<\infty
$$


ЗАмечАнИЕ 3. При $q=\infty$ нужны обычные в этом случае изменения. Далее, $L_{p}\left(\mathbb{R}^{n}\right)$ при $0<p \leqslant \infty$ - обычные пространства, квазинормированные по формуле (11). Пространства $B_{p q}^{s}\left(\mathbb{R}^{n}\right)$ и $F_{p q}^{s}\left(\mathbb{R}^{n}\right)$ квазибанаховы, они не зависят от $\varphi$ (квазинормы эквивалентны). По поводу частных случаев (классических соболевских пространств и соболевских пространств дробного порядка, классических пространств Никольского-Бесова, пространств Харди) см. упомянутую вьше литературу. Заметим только, что

$$
\mathscr{C}^{\sigma}\left(\mathbb{R}^{n}\right)=B_{\infty \infty}^{\sigma}\left(\mathbb{R}^{n}\right), \quad \sigma \in \mathbb{R},
$$

- пространства Гёльдера-Зигмунда (продолженные в область $\sigma \leqslant 0$ ).

2.2. Кварковые разложения и фреймы всплесков. Пусть $\chi_{j m}$, где $j \in \mathbb{N}_{0}$ и $m \in \mathbb{Z}^{n}$, - характеристические функции кубов $Q_{j m}$ в $\mathbb{R}^{n}$ со сторонами, параллельными осям координат, с центрами в $2^{-j} m$ и с длинами сторон $2^{-j+1}$.

ОПРЕДЕЛЕНИЕ 4 . Пусть $\varrho \geqslant 0, s \in \mathbb{R}, 0<p \leqslant \infty, 0<q \leqslant \infty$ и

$$
\lambda=\left\{\lambda_{j m}^{\beta} \in \mathbb{C}: \beta \in \mathbb{N}_{0}^{n}, j \in \mathbb{N}_{0}, m \in \mathbb{Z}^{n}\right\} .
$$

Тогда

$$
\left\|\lambda \mid b_{p q}^{s, \varrho}\right\|=\sup _{\beta \in \mathbb{N}_{0}^{n}} 2^{\varrho|\beta|}\left(\sum_{j=0}^{\infty} 2^{j(s-n / p) q}\left(\sum_{m \in \mathbb{Z}^{n}}\left|\lambda_{j m}^{\beta}\right|^{p}\right)^{q / p}\right)^{1 / q}
$$

и

$$
\left\|\lambda\left|f_{p q}^{s, \varrho}\left\|=\sup _{\beta \in \mathbb{N}_{0}^{n}} 2^{\varrho|\beta|}\right\|\left(\sum_{j=0}^{\infty} \sum_{m \in \mathbb{Z}^{n}} 2^{j s q}\left|\lambda_{j m}^{\beta}\right|{ }^{q} \chi_{j m}(\cdot)\right)^{1 / q}\right| L_{p}\left(\mathbb{R}^{n}\right)\right\|
$$

с обьчньми изменениями при $p=\infty$ и/или $q=\infty$.

ЗАмЕчАнИЕ 5. Соответствуюшие пространства последовательностей $b_{p q}^{s, \varrho}$ и $f_{p q}^{s, \varrho}$ играют определенную роль в теории кварковых разложений функциональных пространств. В частности, мы отсылаем читателя к $\S 2$ в [30]. По происхождению эти пространства связаны с атомическими разложениями пространств функций. Ссылки и пояснения можно найти в $[28 ; \S 1]$ и $[35 ; \S 1]$, а также в [29].

Опишем теперь основные составные части кварковых разложений пространств $B_{p q}^{s}\left(\mathbb{R}^{n}\right)$ и $F_{p q}^{s}\left(\mathbb{R}^{n}\right)$.

ОПреДЕЛЕниЕ 6 . Пусть $\omega-$ функция из $C^{\infty}$ на $\mathbb{R}^{n}$ такая, что

$$
\operatorname{supp} \omega \subset(-\pi, \pi)^{n}, \quad \omega(x)=1 \text { при }|x| \leqslant 2 .
$$

Для $J \in \mathbb{N}$, как в (1)-(3), положим

и

$$
\omega^{\beta}(x)=\frac{i^{|\beta|} 2^{J|\beta|}}{(2 \pi)^{n} \beta !} x^{\beta} \omega(x), \quad x \in \mathbb{R}^{n}, \quad \beta \in \mathbb{N}_{0}^{n},
$$

$$
\Omega^{\beta}(x)=\sum_{m \in \mathbb{Z}^{n}}\left(\omega^{\beta}\right)^{\vee}(m) e^{-i m x}, \quad x \in \mathbb{R}^{n}, \quad \beta \in \mathbb{N}_{0}^{n} .
$$

Пусть $\varphi_{0}$ и $\varphi_{1}-$ такие же, как в (12). Тогда для $\beta \in \mathbb{N}_{0}^{n}$ и $m \in \mathbb{Z}^{n}$ имеем

и

$$
\begin{aligned}
\left(\Phi_{F}^{\beta}\right)^{\vee}(\xi) & =\varphi_{0}(\xi) \Omega^{\beta}(\xi), & & \xi \in \mathbb{R}^{n}, \\
\left(\Phi_{M}^{\beta}\right)^{\vee}(\xi) & =\varphi_{1}(\xi) \Omega^{\beta}(\xi), & & \xi \in \mathbb{R}^{n},
\end{aligned}
$$

$$
\Phi_{j m}^{\beta}(x)= \begin{cases}\Phi_{F}^{\beta}(x-m), & \text { если } j=0, \\ \Phi_{M}^{\beta}\left(2^{j} x-m\right), & \text { если } j \in \mathbb{N} .\end{cases}
$$


ЗАмЕчАНИЕ 7. Мы следовали [31] с небольшими изменениями в соответствии с [35; пा. 3.2.1 и 3.2.2]. Там же можно найти и дальнейшие пояснения. В частности,

$$
\omega^{\beta}(x)=(2 \pi)^{-n / 2} \Omega^{\beta}(x), \text { если } x \in(-\pi, \pi)^{n} .
$$

Следовательно, $\Phi_{F}^{\beta}, \Phi_{M}^{\beta}$ и $\Phi_{j m}^{\beta}$ - аналитические функции, принадлежашие $S\left(\mathbb{R}^{n}\right)$. Кроме того, согласно замечанию 3.22 в [35], существует такая постоянная $c \geqslant 0$, что

$$
\left|D^{\alpha}\left[\xi^{\beta} \omega(\xi)\right]\right| \leqslant c_{K}(1+|\beta|)^{2 K} 2^{c|\beta|}
$$

для любых $K \in \mathbb{N}$, любых $\alpha \in \mathbb{N}_{0}^{n}$ с $|\alpha| \leqslant 2 K$, любых $\beta \in \mathbb{N}_{0}^{n}$, любых $\xi \in \mathbb{R}^{n}$ и некоторой постоянной $c_{K} \cdot$ И (18), и (19) будут нужны нам в дальнейшем. Эти неравенства могут быть также получены элементарными рассуждениями. Пусть $\sigma_{p}$ и $\sigma_{p q}-$ те же числа, что в (9) и (10). Пусть

$$
\lambda_{j m}^{\beta}(f)=2^{j n}\left(f, \Phi_{j m}^{\beta}\right) \text { при } f \in S^{\prime}\left(\mathbb{R}^{n}\right) .
$$

Пусть $\bar{p}=p$ при $1 \leqslant p \leqslant \infty$ и $\bar{p}=1$ при $0<p<1$. Пусть $L_{\infty}\left(\mathbb{R}^{n}, w_{\sigma}\right)$ - очевидным образом нормированное пространство всех функций $f$, удовлетворяюших условию $w_{\sigma} f \in L_{\infty}\left(\mathbb{R}^{n}\right)$, где $w_{\sigma}(x)=\left(1+|x|^{2}\right)^{\sigma / 2}$. В следуюшей теореме обсуждаются представления вида (8), где $\beta \in \mathbb{N}_{0}^{n}, j \in \mathbb{N}_{0}, m \in \mathbb{Z}^{n}$. В любом случае соответствующий ряд сходится абсолютно (и поэтому безусловно) в $L_{\bar{p}}\left(\mathbb{R}^{n}\right)$ при $p<\infty$ и в $L_{\infty}\left(\mathbb{R}^{n}, w_{\sigma}\right)$ с $\sigma<0$ при $p=\infty$. Это оправдьвает запись и в $(8)$, и в дальнейшем.

TеОрема 8. Пусть $k_{j m}^{\beta}$ (me жее, что в (1)-(4)) u $\Phi_{j m}^{\beta}($ как в определении 6$)$ - две фиксированные системьл. Пусть $\varrho \geqslant 0 u 0<q \leqslant \infty$.

(i) Пусть $0<p \leqslant \infty$ и $>>\sigma_{p}$. Тогда $f \in S^{\prime}\left(\mathbb{R}^{n}\right)$ является әлементом $B_{p q}^{s}\left(\mathbb{R}^{n}\right)$ в том и только том случае, если $f$ допускает представление в виде

$$
f=\sum_{\beta, j, m} \lambda_{j m}^{\beta} k_{j m}^{\beta}, \quad \lambda \in b_{p q}^{s, \varrho},
$$

причем ряд абсолютно сходится в $L_{\bar{p}}\left(\mathbb{R}^{n}\right)$ при $p<\infty u$ в $L_{\infty}\left(\mathbb{R}^{n}, w_{\sigma}\right)$, где $\sigma<0$, при $p=\infty$. Кроме того,

$$
\left\|f\left|B_{p q}^{s}\left(\mathbb{R}^{n}\right)\|\sim \inf \| \lambda\right| b_{p q}^{s, \varrho}\right\|
$$

- эквивалентные квазинормы, если нижняя грань берется по множеству всех допустимых представлений (21).

(ii) Пусть $0<p<\infty u s>\sigma_{p q}$. Тогда $f \in S^{\prime}\left(\mathbb{R}^{n}\right)$ является әлементом $F_{p q}^{s}\left(\mathbb{R}^{n}\right)$ в том и только том случае, если $f$ может быть представлена в виде

$$
f=\sum_{\beta, j, m} \lambda_{j m}^{\beta} k_{j m}^{\beta}, \quad \lambda \in f_{p q}^{s, \varrho},
$$

причем ряд абсолютно сходится в $L_{\bar{p}}\left(\mathbb{R}^{n}\right)$. Кроме того,

$$
\left\|f\left|F_{p q}^{s}\left(\mathbb{R}^{n}\right)\|\sim \inf \| \lambda\right| f_{p q}^{s, \varrho}\right\|
$$

- эквивалентные квазинормы, где нижняя грань берется по множеству всех допустимых представлений (22). 
(iii) Пусть $\lambda_{j m}^{\beta}(f)$ заданьь формулой (20). Пусть $0<p \leqslant \infty u s>\sigma_{p}$. Тогда $f \in B_{p p}^{s}\left(\mathbb{R}^{n}\right)$ можкно представить в виде

$$
f=\sum_{\beta, j, m} \lambda_{j m}^{\beta}(f) k_{j m}^{\beta}
$$

причем ряд абсолютно сходится в $L_{\bar{p}}\left(\mathbb{R}^{n}\right)$ при $p<\infty$ и в $L_{\infty}\left(\mathbb{R}^{n}, w_{\sigma}\right)$ для $\sigma<0$ при $p=\infty$. Кроме того, $\lambda(f) \in b_{p p}^{s, \varrho} u$

$$
\left\|f\left|B_{p p}^{s}\left(\mathbb{R}^{n}\right)\|\sim\| \lambda(f)\right| b_{p p}^{s, \varrho}\right\|
$$

( эквивалентные квазинормы).

ЗАмЕчАниЕ 9. Утверждения (i) и (ii) - частные случаи более общих теорем о кварковых разложениях из [30; $§ 2]$, которые также приведены (без доказательств) в [35; $\S 1.6]$. Утверждение (iii) содержится в [31] и [35; 3.2 .2$]$. Добавим два замечания. Во-первых, можно спросить, для всех ли пространств $B_{p q}^{s}\left(\mathbb{R}^{n}\right)$, указанных в (i), и всех ли пространств $F_{p q}^{s}\left(\mathbb{R}^{n}\right)$, указанных в (ii), сушествуют оптимальные базисные представления, как в (23), удовлетворяющие условиям (20) и (24). Это возможно установить, но требует некоторых дополнительных усилий. Сошлемся на [30; следствие 2.12]. Во-вторых, в вышеприведенных утверждениях о сходимости можно заменить $L_{\bar{p}}$ любьм пространством

$$
L_{r} \quad \text { с } \quad p \leqslant r \leqslant \infty \quad \text { и } \frac{1}{r}>\frac{1}{p}-\frac{s}{n} .
$$

Этот факт следует из хорошо известных вложений. Выбор $r=\bar{p} \geqslant 1$ проясняет, что все происходит в рамках пространства $S^{\prime}\left(\mathbb{R}^{n}\right)$. Для наших дальнейших целей выбор $r=p$, и потому абсолютная сходимость в $L_{p}\left(\mathbb{R}^{n}\right)$ (с указанными изменениями при $p=\infty)$, лучше приспособлен.

\section{§ 3. Доказательство теоремы 1 , дальнейшие комментарии}

\section{1. Доказательство теоремы 1.}

Шаг 1. Докажем сначала утверждения (5) и (6) с $r=0$ и $l=0$. Напомним, что $K^{\gamma}(x)=\left(2^{-J} x\right)^{\gamma} K(x)$ с $\gamma \in \mathbb{N}_{0}^{n}$. Согласно (23), (20) и (17), имеем

$$
\begin{gathered}
K^{\gamma}(x)=\sum_{\beta, j, m} \lambda_{j m}^{\beta}\left(K^{\gamma}\right) k_{j m}^{\beta}(x), \\
\lambda_{j m}^{\beta}\left(K^{\gamma}\right)=2^{j n} \int_{\mathbb{R}^{n}} K^{\gamma}(y) \Phi_{M}^{\beta}\left(2^{j} y-m\right) d y
\end{gathered}
$$

при $j \in \mathbb{N}$. Если $j=0$, то здесь и в дальнейшем нужны соответствуюшие изменения. Пусть $\alpha \in \mathbb{N}_{0}^{n}$ с $|\alpha| \leqslant 2 A$ при некотором $A \in \mathbb{N}$. Тогда из (16) следует (на основании $(18),(15),(19)$ и формулы Стирлинга для $N$ !), что

$$
\left|D^{\alpha}\left(\Phi_{M}^{\beta}\right)^{\vee}(\xi)\right| \leqslant c_{A, \varkappa} 2^{-\varkappa|\beta|}
$$

для любого $\varkappa>0$ и любого $\beta \in \mathbb{N}_{0}^{n}$. Из свойств носителя функции $\varphi_{1}$ из (12) и стандартных утверждений о преобразовании Фурье следует, что, с одной стороны, для любого $b>0$, любого $\varkappa>0$ и любого $\beta \in \mathbb{N}_{0}^{n}$ имеем

$$
\left|\Phi_{M}^{\beta}(y)\right| \leqslant c_{b, \varkappa} 2^{-\varkappa|\beta|}(1+|y|)^{-b}, \quad y \in \mathbb{R}^{n},
$$


и, с другой стороны,

$$
\left(\Phi_{M}^{\beta}\right)^{\vee}(\xi)=|\xi|^{2 L} \frac{\varphi_{1}(\xi)}{|\xi|^{2 L}} \Omega^{\beta}(\xi)=|\xi|^{2 L} \widetilde{\Omega}^{\beta}(\xi)
$$

для любого $L \in \mathbb{N}$, и поэтому

$$
\Phi_{M}^{\beta}(y)=\Delta^{L} \widetilde{\Phi}_{M}^{\beta}(y), \quad y \in \mathbb{R}^{n},
$$

где $\Delta^{L}-L$-я степень лапласиана $\Delta$. Здесь $\widetilde{\Phi}_{M}^{\beta}$ имеет те же свойства, что и $\Phi_{M}^{\beta}$. В частности, формула (27) выполняется с $\widetilde{\Phi}_{M}^{\beta}$ вместо $\Phi_{M}^{\beta}$. Тогда, интегрируя по частям, видим, что

$$
\int_{\mathbb{R}^{n}}\left(\Delta^{L} K^{\gamma}\right)(y) \widetilde{\Phi}_{M}^{\beta}\left(2^{j} y-m\right) d y=2^{2 j L} \int_{\mathbb{R}^{n}} K^{\gamma}(y) \Phi_{M}^{\beta}\left(2^{j} y-m\right) d y .
$$

Из $(26)$ и $(27)$ с $\widetilde{\Phi}$ вместо $\Phi$ и свойств $K^{\gamma}$ получаем

$$
\begin{aligned}
\left|\lambda_{j m}^{\beta}\left(K^{\gamma}\right)\right| & \leqslant c 2^{j n-2 j L} 2^{-\varepsilon|\gamma|} 2^{-\varkappa|\beta|} \int_{|y| \leqslant 2^{J}}\left(1+\left|2^{j} y-m\right|\right)^{-b} d y \\
& \leqslant c^{\prime} 2^{j(n+b-2 L)} 2^{-\varepsilon|\gamma|} 2^{-\varkappa|\beta|}(1+|m|)^{-b} .
\end{aligned}
$$

Так как $b>0$, то $L \in \mathbb{N}$ и $\varkappa>0$ - в нашем распоряжении, а формулы (25) и (28) доказывают (5) и (6) с $r=0$ и $l=0$, и поэтому

$$
\left|\lambda_{j m}^{\gamma}\left(K^{\gamma}\right)\right| \leqslant c 2^{-\varepsilon|\gamma|} 2^{-\varkappa|\beta|} 2^{-j M}(1+|m|)^{-b}
$$

Шаг 2. Пусть $r=0$ и $l \in \mathbb{Z}^{n}$. Тогда из (25) следует, что

$$
K_{0, l}^{\gamma}(x)=K^{\gamma}(x-l)=\sum_{\beta, j, m} \lambda_{j m}^{\beta}\left(K^{\gamma}\right) k_{j, m+2^{j} l}^{\beta}(x)=\sum_{\beta, j, m} \nu_{j m}^{\beta}\left(K_{0, l}^{\gamma}\right) k_{j m}^{\beta}(x)
$$

$\mathrm{c} \nu_{j m}^{\beta}\left(K_{0, l}^{\gamma}\right)=\lambda_{j, m-2^{j} l}^{\beta}\left(K^{\gamma}\right)$. Из $(29)$ получаем

$$
\left|\nu_{j m}^{\beta}\left(K_{0, l}^{\gamma}\right)\right| \leqslant c 2^{-\varepsilon|\gamma|} 2^{-\varkappa|\beta|} 2^{-j M}\left(1+\left|m-2^{j} l\right|\right)^{-b},
$$

откуда следует (6) с $r=0$. Пусть $r \in \mathbb{N}$ и $l \in \mathbb{Z}^{n}$. Тогда из (30) выводим

$$
K_{r l}^{\gamma}(x)=K^{\gamma}\left(2^{r} x-l\right)=\sum_{\beta, j, m} \nu_{j m}^{\beta}\left(K_{0, l}^{\gamma}\right) k_{j+r, m}^{\beta}(x)=\sum_{\beta, j \geqslant r, m} \mu_{j m}^{\beta}\left(K_{r l}^{\gamma}\right) k_{j m}^{\beta}(x)
$$

с $\mu_{j m}^{\beta}\left(K_{r l}^{\gamma}\right)=\nu_{j-r, m}^{\beta}\left(K_{0, l}^{\gamma}\right)$. Из (31) получаем (6).

Шаг 3. Согласно (20) и приведенному вьше сдвигу в индексах из $\mathbb{Z}^{n}$ и $\mathbb{N}_{0}$, коэффициенты $\mu_{j m}^{\beta}\left(K_{r l}^{\gamma}\right)$ в $(32)$ можно представить в виде $(5)$ с некоторыми $\Psi_{j, r ; m, l}^{\beta, \gamma} \in S\left(\mathbb{R}^{n}\right)$, которые можно вычислить явно. Согласно $(5),(6)$ и свойствам $k_{j m}^{\beta}$, ряд в $(5)$ сходится в норме

$$
\sup _{x \in \mathbb{R}^{n},|\alpha| \leqslant L}\left(1+|x|^{2}\right)^{\sigma / 2}\left|D^{\alpha} g(x)\right|
$$

с любыми $\sigma>0$ и $L \in \mathbb{N}$. Следовательно, ряд (5) сходится безусловно в $S\left(\mathbb{R}^{n}\right)$. 


\section{2. Свойство мультиразрешения.}

ЗАмЕчАниЕ 10. Возникает вопрос: какие свойства функций $k$ и $K$ в теореме 1 действительно нужны, чтобы получить утверждение типа $(5),(6)$ ? Что касается $k$, то и вьшеприведенные рассуждения, и базисное представление в теореме 8 (iii) справедливы для любого разложения единицы с условием (2), основанного на финитной бесконечно дифференцируемой функции $k$ на $\mathbb{R}^{n}$. (Некоторые технические изменения при этом необходимы.) Что же касается $K$, то мы используем только тот факт, что $K-$ финитная бесконечно дифференцируемая функция. Но $C^{\infty}$-условие можно ослабить до $C^{N}$-условия, а условие, что $K$ - финитная функция, до соответствующего условия достаточно быстрого убывания $K$ (в зависимости от имеюшихся в виду приложений).

ЗАмЕчАниЕ 11 . Недавно развитая теория ортогональных базисов всплесков, скажем, в $L_{2}(\mathbb{R})$, обычно основывается на так называемом анализе мультиразложсений с типичным утверждением

$$
\Phi(x)=\sum_{m=-\infty}^{\infty} a_{m} \Phi(2 x-m), \quad x \in \mathbb{R},
$$

для масштабирующей функции $\Phi$, где $a_{m}$ - подходящие комплексные числа (см., например, [37]). По теореме 1 и в силу высказанных вьше замечаний получаем следующее дополнение.

СледСТВИЕ 12. Пусть $k$ - финитная бесконечно дифференцируемая функция на $\mathbb{R}^{n}$, порождающая разложсние единиць (2). Пусть $k_{\beta}(x)=x^{\beta} k(x)$, где $\beta \in \mathbb{N}_{0}^{n}$. Пусть $N \in \mathbb{N}$. Тогда существуют такие комплекснье числа $\lambda_{j m}^{\beta}$, чmo

$$
k(x)=\sum_{\beta \in \mathbb{N}_{0}^{n}} \sum_{j=N}^{\infty} \sum_{m \in \mathbb{Z}^{n}} \lambda_{j m}^{\beta} k_{\beta}\left(2^{j} x-m\right), \quad x \in \mathbb{R}^{n},
$$

где ряд безусловно сходится в $S\left(\mathbb{R}^{n}\right)$. Для любих $\varkappa>0, M>0 u b>0$ существует положительная постоянная $c=c_{\varkappa, M, b}($ зависящая от $k$ u $N)$ такая, что

$$
\left|\lambda_{j m}^{\beta}\right| \leqslant c 2^{-\varkappa|\beta|} 2^{-j M}(1+|m|)^{-b} .
$$

ДокАЗАТЕЛЬСТВо. Это утверждение следует из рассуждений, примененных на шаге 1 в п.3.1, комментариев в замечании 10 и формулы (29).

ЗАМЕЧАНИЕ 13. Можно говорить о свойстве мультиразрешения, если грубые сетки $k(x-m)$ или $k^{\beta}(x-m)$ можно заменить более тонкими сетками $k_{j m}^{\beta}, j \in \mathbb{N}$, порожденными той же функцией $k$.

\section{$\S 4$. Пространства на $\mathbb{R}^{n}$}

4.1. Определение и основные утверждения. Теорема 1 - краеугольный камень всего последуюшего изложения. Пространства последовательностей $b_{p q}^{s, \varrho}$ и $f_{p q}^{s, \varrho}$ имеют здесь тот же смысл, что в определении 4 и замечании 5. Кроме того, $L_{p}\left(\mathbb{R}^{n}\right)$ - обычные лебеговские пространства, квазинормированные по формуле (11), и $L_{\infty}\left(\mathbb{R}^{n}, w_{\sigma}\right)$ определено в замечании 7 .

ОПРЕДЕЛЕНИЕ 14. Пусть $k$ - неотрицательная бесконечно дифференцируемая функция на $\mathbb{R}^{n}$, удовлетворяющая условию (1) при некотором $J \in \mathbb{N}$ (мы можем фиксировать $J=n$ ) и условию (2). Пусть $k_{j m}^{\beta}$ как в (4). Пусть $\varrho \geqslant 0,0<q \leqslant \infty$ и $s>0$. 
(i) Пусть $0<p \leqslant \infty$. Тогда $\mathfrak{B}_{p q}^{s}\left(\mathbb{R}^{n}\right)$ - семейство всех функций $f \in L_{p}\left(\mathbb{R}^{n}\right)$, которые могут быть представлены в виде

$$
f=\sum_{\beta, j, m} \lambda_{j m}^{\beta} k_{j m}^{\beta}, \quad \lambda \in b_{p q}^{s, \varrho},
$$

и ряд абсолютно сходится в $L_{p}\left(\mathbb{R}^{n}\right)$ при $p<\infty$ и в $L_{\infty}\left(\mathbb{R}^{n}, w_{\sigma}\right)$ для $\sigma<0$ при $p=\infty$. Пусть

$$
\left\|f\left|\mathfrak{B}_{p q}^{s}\left(\mathbb{R}^{n}\right)\|=\inf \| \lambda\right| b_{p q}^{s, \varrho}\right\|,
$$

где нижняя грань берется по всем допустимым представлениям функции $f$ в виде (33).

(ii) Пусть $0<p<\infty$. Тогда $\mathfrak{F}_{p q}^{s}\left(\mathbb{R}^{n}\right)$ - семейство всех функций $f \in L_{p}\left(\mathbb{R}^{n}\right)$, которые могут быть представлены в виде

$$
f=\sum_{\beta, j, m} \lambda_{j m}^{\beta} k_{j m}^{\beta}, \quad \lambda \in f_{p q}^{s, \varrho},
$$

и ряд абсолютно сходится в $L_{p}\left(\mathbb{R}^{n}\right)$. Пусть

$$
\left\|f\left|\mathfrak{F}_{p q}^{s}\left(\mathbb{R}^{n}\right)\|=\inf \| \lambda\right| f_{p q}^{s, \varrho}\right\|,
$$

где нижняя грань берется по всем допустимым представлениям функции $f$ в виде (34).

ЗАмЕчАниЕ 15 . Чтобы избежать недопонимания, заметим, что абсолютная сходимость ряда (33) вытекает из условия $\lambda \in b_{p q}^{s, \varrho}$ и не является дополнительньм требованием. Ситуация с рядом (34) аналогична.

ТЕОРема 16. Введенные выше пространства $\mathfrak{B}_{p q}^{s}\left(\mathbb{R}^{n}\right)$ и $\mathfrak{F}_{p q}^{s}\left(\mathbb{R}^{n}\right)$ являются квазибанаховыми. Они не зависят от $е к$ (квазинормы әквивалентны). Кроме того, для всех допустимых $s, p$ и q имеем

$$
\mathfrak{B}_{p q}^{s}\left(\mathbb{R}^{n}\right) \hookrightarrow L_{p}\left(\mathbb{R}^{n}\right), \quad \mathfrak{F}_{p q}^{s}\left(\mathbb{R}^{n}\right) \hookrightarrow L_{p}\left(\mathbb{R}^{n}\right),
$$

$$
\mathfrak{B}_{p, \min (p, q)}^{s}\left(\mathbb{R}^{n}\right) \hookrightarrow \mathfrak{F}_{p q}^{s}\left(\mathbb{R}^{n}\right) \hookrightarrow \mathfrak{B}_{p, \max (p, q)}^{s}\left(\mathbb{R}^{n}\right) .
$$

ДокАЗАТЕльСтво. Шаг 1 . Пусть $\varrho \geqslant 0$ фиксировано. Непрерьвное вложение (36) следует из соответствующего включения для самих пространств последовательностей $[29 ;$ с. 75 , предложение 13.6]. Докажем наличие непрерьвных вложений (35). Согласно (36), достаточно обсудить первое включение. Пусть $0<p \leqslant 1$ (иначе нужны соответствуюшие изменения). Тогда из (1), (3) и (14) следует, что

$$
\begin{aligned}
\left\|\sum_{\beta, j, m} \lambda_{j k}^{\beta} k_{j m}^{\beta} \mid L_{p}\left(\mathbb{R}^{n}\right)\right\|^{p} & \leqslant \sum_{\beta, j} \int_{\mathbb{R}^{n}}\left|\sum_{m} \lambda_{j m}^{\beta} k^{\beta}\left(2^{j} x-m\right)\right|^{p} d x \\
& \leqslant c \sum_{\beta, j} 2^{-j n} 2^{-\varepsilon|\beta| p} \sum_{m}\left|\lambda_{j m}^{\beta}\right|^{p} \leqslant c^{\prime} \sum_{\beta, j} 2^{-\varepsilon|\beta| p-j s p} .
\end{aligned}
$$

Это доказьвает (35) (мы использовали тот факт, что $\varepsilon>0$ и $s>0$ ). Тогда из стандартных рассуждений следует, что $\mathfrak{B}_{p q}^{s}\left(\mathbb{R}^{n}\right)$ и $\mathfrak{F}_{p q}^{s}\left(\mathbb{R}^{n}\right)$ - квазибанаховы пространства.

Шаг 2. Остается доказать независимость результата от $\varrho$ и $k$. Обсудим сначала случай $\mathfrak{B}$-пространств. Предположим, что $f$ задана вариантом формулы (33) с $K$ вместо $k$, где $K$ имеет тот же смысл, что и в теореме 1 . Тогда

$$
f(x)=\sum_{\gamma, r, l} \nu_{r l}^{\gamma} K_{r l}^{\gamma}(x), \quad \nu \in b_{p q}^{s, \varrho} .
$$


Подставляя (5), переформулированное как (32), в (38), получаем

$$
f(x)=\sum_{\beta, j, m} \lambda_{j m}^{\beta} k_{j m}^{\beta}(x),
$$

где

$$
\lambda_{j m}^{\beta}=\sum_{\gamma \in \mathbb{N}_{0}^{n}} \sum_{r=0}^{j} \sum_{l \in \mathbb{Z}^{n}} \nu_{r l}^{\gamma} \cdot \mu_{j m}^{\beta}\left(K_{r l}^{\gamma}\right) .
$$

Пусть $\nu_{r l}^{\gamma}=0$, если $r<0$, и $t=j-r$. Тогда из (6) (с $\mu$-коэффициентами в левой части) вытекает, что

$$
\begin{aligned}
& 2^{j(s-n / p)} 2^{\varkappa|\beta|}\left|\lambda_{j m}^{\beta}\right| \\
& \quad \leqslant c \sum_{\gamma} 2^{-\varepsilon|\gamma|} \sum_{t=0}^{\infty} \sum_{l \in \mathbb{Z}^{n}} 2^{(j-t)(s-n / p)}\left|\nu_{j-t, l}^{\gamma}\right| 2^{-t M^{\prime}}\left(1+\left|m-2^{t} l\right|\right)^{-b} \\
& \quad \leqslant c^{\prime} \sum_{\gamma} 2^{-\varepsilon|\gamma|}\left(\sum_{t, l} 2^{(j-t)(s-n / p) p}\left|\nu_{j-t, l}^{\gamma}\right|^{p} 2^{-t M^{\prime \prime} p}\left(1+\left|m-2^{t} l\right|\right)^{-b^{\prime} p}\right)^{1 / p},
\end{aligned}
$$

где $\varkappa>0, M^{\prime \prime}>0$ и $b^{\prime}>0$ - в нашем распоряжении. Возьмем $\ell_{p}$-квазинорму относительно $m \in \mathbb{Z}^{n}$. Соответствуюшие множители в правой части (40) можно оценить независимо от $2^{t} l$ (если выбрать $b^{\prime}>0$ достаточно большим). Тогда суммирование по $l \in \mathbb{Z}^{n}$ дает требуемые $\ell_{p}$-блоки для $\nu_{j-t, l}^{\gamma}$-коэффициентов. Затем можно сделать то же самое относительно $\ell_{q}$ и суммирования относительно $j$ за счет $M^{\prime \prime}>0$. Так как $\varepsilon>0$, то

$$
\left\|\lambda\left|b_{p q}^{s, \varkappa}\|\leqslant c\| \nu\right| b_{p q}^{s, 0}\right\| \leqslant c\left\|\nu \mid b_{p q}^{s, \varrho}\right\|
$$

для любого $\varrho \geqslant 0$ и любого $\varkappa \geqslant 0$. Это доказьвает независимость пространств $\mathfrak{B}_{p q}^{s}\left(\mathbb{R}^{n}\right)$ и от $\varrho$, и от $k$.

Шаг 3. Докажем, что $\mathfrak{F}$-пространства не зависят от $\varrho$ и $k$. Нам нужна некоторая подготовка. Напомним, что для любой локально интегрируемой функции $g$ на $\mathbb{R}^{n}$ функция

$$
(M g)(x)=\sup |Q|^{-1} \int_{Q}|g(y)| d y, \quad x \in \mathbb{R}^{n},
$$

где верхняя грань берется по всем кубам $Q$ с центром в $x$, назьвается максимальной функцией Харди-Литлвуда.. Пусть $0<p<\infty, 0<q \leqslant \infty$ и $0<w<\min (p, q)$. Тогда сушествует такая постоянная $c$, что

$$
\left\|\left(\sum_{j, m}\left(M\left|g_{j m}\right|^{w}\right)(\cdot)^{q / w}\right)^{1 / q}\left|L_{p}\left(\mathbb{R}^{n}\right)\|\leqslant c\|\left(\sum_{j, m}\left|g_{j m}(\cdot)\right|^{q}\right)^{1 / q}\right| L_{p}\left(\mathbb{R}^{n}\right)\right\|
$$

для всех функций $g_{j m}$ рассматриваемого класса с $j \in \mathbb{N}_{0}$ и $m \in \mathbb{Z}^{n}$. Это векторнозначное "максимальное неравенство" восходит к [11]. Короткое доказательство приведено в [26; с. $303-305]$. По поводу применения неравенства $(43)$ в контексте $F$-пространств и дальнейших ссылок мы отсылаем читателя к [28; с. 89], и [29; с. 79]. Теперь нам нужна следуюшая подготовка. Пусть $\chi_{r}-$ характеристическая функция куба со стороной $2^{-r}$ при $r \in \mathbb{N}_{0}$ и центром в начале координат. Тогда из (42) следует неравенство

$$
\left(M \chi_{r}\right)(x) \geqslant c \min \left[1,2^{-r n}|x|^{-n}\right], \quad x \in \mathbb{R}^{n},
$$


для некоторого $c>0$ (не зависяшего от $r$ ). Сравним $\chi_{j m}$ с $M \chi_{r l}$, как это требуется в $(39)$, в связи с $f_{p q}^{s, \varrho}$ (в соответствии с определением 4$)$. В частности, $r \leqslant j$. Тогда для $x=2^{-j} m$ из (44) получаем

$$
\left(M_{\chi_{r l}}\right)(x)^{1 / w} \geqslant c \min \left[1,\left(2^{-r n}\left|2^{-j} m-2^{-r} l\right|^{-n}\right)^{1 / w}\right] \geqslant c\left[1,\left|m-2^{j-r} l\right|^{-n / w}\right] .
$$

Эта оценка остается справедливой для всех $x \in \mathbb{R}^{n}$ с $\chi_{j m}(x)=1$ в левой части формулы (45) и той же правой частью. При $t=j-r$ получаем

$$
\chi_{j m}(x) \leqslant c^{\prime}\left(1+\left|m-2^{t} l\right|\right)^{n / w}\left(M \chi_{j-t, l}\right)(x)^{1 / w}, \quad x \in \mathbb{R}^{n} .
$$

Умножим (39) на $\chi_{j m}(x)$ и применим (46). Так как $b>0$ в (40) находится в нашем распоряжении, мы приходим к варианту формулы (41) с $q$ вместо $p$. Применяя (43), получаем $f$-вариант формулы (41). Это завершает доказательство.

ЗАмечАниЕ 17 . Тот факт, что $\varrho$ в определении 14 и теореме 16 можно взять сколь угодно большим (эквивалентные квазинормы), вьплядит почти черной магией. Это следует из (40) и, как следствие, из (41) с $\varkappa=\varrho$. Для лучшего понимания можно выяснить зависимость величины $c$ в $(6)$ от $\varkappa=\varrho$, что, в свою очередь, непосредственно влияет на постоянные в эквивалентных квазинормах для различных значений $\varrho$. В несколько ином, но близком контексте мы оцениваем соответствующие постоянные в [30; $(2.81)$, с. 23]. Это наводит на мысль, что $c$ в (6) можно заменить на $c_{1} 2^{c_{2} \varkappa}$, где $c_{1}>0$ и $c_{2}>0$ не зависят от $\varkappa$. Следовательно, цена, которую нужно заплатить за быстрое убьвание $2^{-\varkappa|\beta|}-$ это экспоненциально взрывающиеся постоянные $c_{1} 2^{c_{2} \varkappa}$.

4.2. Свойства. Пусть $\sigma_{p}$ и $\sigma_{p q}$ те же, что в (9) и (10).

ТЕОРема 18. Пусть $B_{p q}^{s}\left(\mathbb{R}^{n}\right)$ и $F_{p q}^{s}\left(\mathbb{R}^{n}\right)$ - те жее, что в определении 2 , и пусть $\mathfrak{B}_{p q}^{s}\left(\mathbb{R}^{n}\right)$ и $\mathfrak{F}_{p q}^{s}\left(\mathbb{R}^{n}\right)$ - те жсе, что в определении 14. Тогда

$$
B_{p q}^{s}\left(\mathbb{R}^{n}\right)=\mathfrak{B}_{p q}^{s}\left(\mathbb{R}^{n}\right) \quad \text { npu } \quad s>\sigma_{p}
$$

$u$

$$
F_{p q}^{s}\left(\mathbb{R}^{n}\right)=\mathfrak{F}_{p q}^{s}\left(\mathbb{R}^{n}\right) \quad \text { npu } \quad s>\sigma_{p q},
$$

если их интерпретировать как подпространства в $S^{\prime}\left(\mathbb{R}^{n}\right)$.

ДокАЗАТЕльство. Это непосредственно следует из теоремы 8 и замечания 9.

ЗАмЕчАНИЕ 19. По теореме 8 и замечанию 9, базисные представления (23) и (20) имеют место для всех пространств в предыдущей теореме. Но неясно, имеет ли место соответствуюшее свойство для $\mathfrak{B}-$ и $\mathfrak{F}$-пространств, не рассматриваемых в предыдущей теореме. Если $0<s<\sigma_{p}$ (и потому $p<1$ ), то ничего подобного формулам (47) и (48) ожидать нельзя. В этом случае пространства $B_{p q}^{s}\left(\mathbb{R}^{n}\right)$ и $F_{p q}^{s}\left(\mathbb{R}^{n}\right)$ содержат сингулярные распределения, например, $\delta$-функцию, в то время как $\mathfrak{B}_{p q}^{s}\left(\mathbb{R}^{n}\right)$ и $\mathfrak{F}_{p q}^{s}\left(\mathbb{R}^{n}\right)$ подмножества $L_{p}\left(\mathbb{R}^{n}\right)$. Но есть и другая возможность. Пусть

$$
\left(\Delta_{h}^{1} f\right)(x)=f(x+h)-f(x), \quad \Delta_{h}^{l+1}=\Delta_{h}^{1} \Delta_{h}^{l},
$$

где $x \in \mathbb{R}^{n}, h \in \mathbb{R}^{n}$ и $l \in \mathbb{N}$, и пусть

$$
\omega_{m}(f, t)_{p}=\sup _{|h| \leqslant t}\left\|\Delta_{h}^{m} f \mid L_{p}\left(\mathbb{R}^{n}\right)\right\|, \quad 0<t<\infty,
$$


- обычньй модуль непрерьвности. Пусть $s>0,0<p \leqslant \infty$ и $0<q \leqslant \infty$. Пусть $s<m \in \mathbb{N}$. Тогда, по определению, $\mathbf{B}_{p q}^{s}\left(\mathbb{R}^{n}\right)$ - это семейство всех $f \in L_{p}\left(\mathbb{R}^{n}\right)$, удовлетворяюших условию

$$
\left\|f\left|\mathbf{B}_{p q}^{s}\left(\mathbb{R}^{n}\right)\left\|_{m}=\right\| f\right| L_{p}\left(\mathbb{R}^{n}\right)\right\|+\left(\int_{0}^{1} t^{-s q} \omega_{m}(f, t)_{p}^{q} \frac{d t}{t}\right)^{1 / q}<\infty
$$

(с обычными изменениями при $q=\infty$ ). Эти пространства не зависят от $m$ (квазинормы эквивалентны); см. [8; гл. 2, §10]. Интерес к этим пространствам вызван теорией приближений и численньм анализом; см. [25] и [9]. Кроме того, можно дополнить формулу (47) следующим образом:

$$
B_{p q}^{s}\left(\mathbb{R}^{n}\right)=\mathbf{B}_{p q}^{s}\left(\mathbb{R}^{n}\right) \text { при } 0<p \leqslant \infty, 0<q \leqslant \infty, s>\sigma_{p},
$$

см. $[28 ; \S 2.6 .1]$. В любом случае пространства $\mathbf{B}_{p q}^{s}\left(\mathbb{R}^{n}\right)$ являются подпространствами $L_{p}\left(\mathbb{R}^{n}\right)$, и можно задать вопрос, верно ли, что

$$
\mathfrak{B}_{p q}^{s}\left(\mathbb{R}^{n}\right)=\mathbf{B}_{p q}^{s}\left(\mathbb{R}^{n}\right) \text { при } 0<p \leqslant \infty, 0<q \leqslant \infty, s>0 .
$$

Для $\mathfrak{F}$-пространств также есть хороший кандидат на $F$-часть в $(50)$. Ю. В. Нетрусов [22] дополнил шкалу $F_{p q}^{s}\left(\mathbb{R}^{n}\right)$ пространствами $\mathbf{F}_{p q}^{s}\left(\mathbb{R}^{n}\right)=F L_{p q}^{s}\left(\mathbb{R}^{n}\right)$ с $s>0$, $0<p<\infty$ и $0<q \leqslant \infty$, как подпространствами в $L_{p}\left(\mathbb{R}^{n}\right)$. В этой связи мы также упомянем недавнюю существенную работу [18], продолжающую [22] и [1; 110$]$, и особенно теорему 1.1.14 в [18]. Там можно найти характеризации пространств $\mathbf{F}_{p q}^{s}\left(\mathbb{R}^{n}\right)$ из $\mathbf{F}$-аналога формулы (49) и в терминах атомов, и с помошью разностей. Хотя некоторые детали нуждаются в проверке, ясно, что одновременно выполняется и соотношение (50), и равенство

$$
\mathfrak{F}_{p q}^{s}\left(\mathbb{R}^{n}\right)=\mathbf{F}_{p q}^{s}\left(\mathbb{R}^{n}\right) \text { при } 0<p<\infty, 0<q \leqslant \infty, s>0 .
$$

В одну сторону это следует из наблюдения, что кварковое представление, в соответствии с определением 14 , можно конвертировать в атомическое разложение в соответствии с [22], [18]. Для доказательства обратного утверждения можно применить воспроизводяшую формулу (5) к любому атому (где теперь $\gamma=0$ ) и рассуждать как в доказательстве теоремы 16. Учитьвая упомянутую характеризацию в терминах разностей, мы получаем и (51), и (50).

ЗАмЕчАнИЕ 20. Для пространств $F_{p q}^{s}\left(\mathbb{R}^{n}\right)$ с $s>\sigma_{p q}$ имеет место формула (48) и характеризация в терминах разностей, [28; теорема 3.5.3, с. 194]. В недавней работе [7] указано, что нельзя ожидать такой характеризации в терминах средних значений разностей, если $\sigma_{p}<s<\sigma_{p q}$ (и поэтому $\left.0<q<p\right)$. Можно также учесть формулировки в [35] (§1.11.8, теорема 1.116 и замечание 1.117). В сочетании с теоремой 3.14 в [18], этот факт подсказывает, что

$$
\mathbf{F}_{p q}^{s}\left(\mathbb{R}^{n}\right) \neq F_{p q}^{s}\left(\mathbb{R}^{n}\right) \text { при } 0<p<\infty, 0<q \leqslant \infty, 0<s<\sigma_{p q}
$$

(как подпространства в $L_{p}\left(\mathbb{R}^{n}\right)$ или - если также $s>\sigma_{p}$ - как подпространства в $\left.S^{\prime}\left(\mathbb{R}^{n}\right)\right)$. Конечно, $0<q<p<\infty$ и $\sigma_{p}<s<\sigma_{p q}$ - наиболее поразительный случай в (52). Сравнивая атомические разложения для $F_{p q}^{s}\left(\mathbb{R}^{n}\right)($ см. [22], [12], [13]) с соответствуюшими разложениями для $\mathbf{F}_{p q}^{s}\left(\mathbb{R}^{n}\right)$ (см. [22] и [18; теорема 1.1.14]), автоматически получаем, что обычные условия сокрашения для атомов в $F_{p q}^{s}\left(\mathbb{R}^{n}\right)$ при $s<\sigma_{p q}$ необходимы. В этой связи можно также отметить [35; $§ 1.5$, теорема 1.19 и замечание 1.20]. Это решает давно стоявшую проблему. 
ОПРЕДЕЛЕНИЕ 21. Говорят, что пространство $A\left(\mathbb{R}^{n}\right)$, соответствующее определению 2 или определению 14, назьвается обладает свойством положительности, если любой элемент $f \in A\left(\mathbb{R}^{n}\right)$ допускает разложение

$$
f=f_{1}-f_{2}+i f_{3}-i f_{4} \quad \text { с } f_{l} \geqslant 0, \quad f_{l} \in A\left(\mathbb{R}^{n}\right),
$$

и

$$
\left\|f\left|A\left(\mathbb{R}^{n}\right)\left\|\sim \sum_{l=1}^{4}\right\| f_{l}\right| A\left(\mathbb{R}^{n}\right)\right\|,
$$

где константы эквивалентности не зависят от $f$.

ЗАмечАниЕ 22. Напомним, что условие $f \geqslant 0$ для $f \in S^{\prime}\left(\mathbb{R}^{n}\right)$ означает, что $f(\varphi) \geqslant 0$ для любой вешественной неотрищательной функции $\varphi \in S\left(\mathbb{R}^{n}\right)$.

ТЕОРема 23. Все пространства $\mathfrak{B}_{p q}^{s}\left(\mathbb{R}^{n}\right)$ и $\mathfrak{F}_{p q}^{s}\left(\mathbb{R}^{n}\right)$, введенные в определении 14, имеют свойство положсительности.

ДокАЗАТЕльство. Разлагая $\lambda_{j m}^{\beta} \in \mathbb{C}$ в (33), мы немедленно получаем это утверждение, поскольку $k_{j m}^{\beta} \geqslant 0$ согласно (3) и (4).

ЗАмЕчАнИЕ 24 . В частности, все пространства $B_{p q}^{s}\left(\mathbb{R}^{n}\right)$ в $(47)$ и все пространства $F_{p q}^{s}\left(\mathbb{R}^{n}\right)$ в (48) имеют свойство положительности. Этот факт известен, см. [33] и $[35 ; \oint 3.3 .2]$, где можно также найти дальнейшие утверждения этого типа. В частности, пространства $B_{p q}^{s}\left(\mathbb{R}^{n}\right)$ и $F_{p q}^{s}\left(\mathbb{R}^{n}\right)$ с $0<p \leqslant \infty(p<\infty$ для $F$-пространств $)$, с $0<q \leqslant \infty$ и $-\infty<s<\sigma_{p}$ не имеют свойства положительности. Отсюда следует, что эти пространства не имеют представления из теоремы 8 (i), (ii).

\section{$\S$ 5. Пространства на множествах}

5.1. Предварительные сведения, пространства последовательностей. Определение 4 для пространств последовательностей и определение 14 для функциональных пространств могут быть распространены на случай множеств $M$ в $\mathbb{R}^{n}$. Два случая представляют особьй интерес. Во-первых, случай, когда $M=\Omega$ - область (= открытое множество) в $\mathbb{R}^{n}$, и, во-вторых, случай, когда $M=\Gamma$ - компактное множество (= фрактал) в $\mathbb{R}^{n}$. Что касается пространств $\mathfrak{B}_{p q}^{s}(M)$ и $\mathfrak{F}_{p q}^{s}(M)$, то всегда нужна соответствуюшая версия формулы (35). В случае $M=\Omega$ получаем пространство $L_{p}(\Omega)$, естественно квазинормированное по формуле

$$
\left\|f \mid L_{p}(\Omega)\right\|=\left(\int_{\Omega}|f(x)|^{p} d x\right)^{1 / p}, \quad 0<p \leqslant \infty,
$$

с очевидными изменениями при $p=\infty$. Если $\Gamma-$ компактное множество в $\mathbb{R}^{n}$, то есть искушение воспользоваться замечательным наблюдением [36], что сушествует радонова мера $\mu$ в $\mathbb{R}^{n}$, удовлетворяющая условию удвоения, такая, что

$$
\operatorname{supp} \mu=\Gamma \text { компактен, } \quad 0<\mu(\Gamma)<\infty .
$$

Но у нас другая точка зрения. Мы предпочитаем такие радоновы меры на $\mathbb{R}^{n}$ (удовлетворяющие или не удовлетворяюшие условию удвоения), что их носитель Г в смысле (53) компактен. Можно спросить, каким пространствам $B_{p q}^{s}\left(\mathbb{R}^{n}\right)$ принадлежат такие меры. Это приводит к довольно точному “отпечатку пальца" меры $\mu$, так называемой характеристике Бесова. Мы подробно изучали ее в [35] и в статьях, там цитируемых. Хотя эта характеристика могла бы быть полезна в контексте настоящей 
статьи, мы ограничимся более грубым случаем, оставляя более тонкие исследования до дальнейших публикаций.

Предположим снова, что $Q_{j m}$, где $j \in \mathbb{N}_{0}$ и $m \in \mathbb{Z}^{n},-$ введенные выше кубы со сторонами, параллельными координатньм осям, с центрами в точках вида $2^{-j} m$ и с длинами сторон $2^{-j+1}$. Напомним, что $\mathscr{C}^{\sigma}\left(\mathbb{R}^{n}\right)$ - пространства Гёльдера-Зигмунда (см. (13)). Тогда $\mu \in \mathscr{C}^{-n}\left(\mathbb{R}^{n}\right)$ для любой радоновой меры $\mu$, удовлетворяющей условию (53). Это утверждение можно уточнить следуюшим образом. Пусть

$$
0<\sigma \leqslant n \text { и } \mu_{j}=\sup _{m \in \mathbb{Z}^{n}} \mu\left(Q_{j m}\right), \quad j \in \mathbb{N}_{0} .
$$

Тогда

$$
\mu \in \mathscr{C}^{-\sigma}\left(\mathbb{R}^{n}\right) \Longleftrightarrow \sup _{j} 2^{j(n-\sigma)} \mu_{j}<\infty .
$$

Мы ссылаемся на [32] или $[35 ; \S 1.12]$. При $M \subset \mathbb{R}^{n}$ (либо область $\Omega$, либо компактное множество Г) мы используем сокращение

$$
\sum_{m}^{M, j}=\sum_{m \in \mathbb{Z}^{n}, Q_{j m} \cap M \neq \varnothing}, \text { где } j \in \mathbb{N}_{0} .
$$

Как и вьше, $\chi_{j m}-$ характеристическая функция $Q_{j m}$.

ОПРЕДЕЛЕНИЕ 25 . Пусть $\varrho \geqslant 0, s \in \mathbb{R}, 0<p \leqslant \infty$ и $0<q \leqslant \infty$. Пусть $M \subset \mathbb{R}^{n}$ и

$$
\lambda=\left\{\lambda_{j m}^{\beta} \in \mathbb{C}: \beta \in \mathbb{N}_{0}^{n}, j \in \mathbb{N}_{0}, m \in \mathbb{Z}^{n} \text { и } Q_{j m} \cap M \neq \varnothing\right\} .
$$

Тогда

$$
\left\|\lambda \mid b_{p q}^{s, \varrho}(M)\right\|=\sup _{\beta \in \mathbb{N}_{0}^{n}} 2^{\varrho|\beta|}\left(\sum_{j=0}^{\infty} 2^{j(s-n / p) q}\left(\sum_{m}^{M, j}\left|\lambda_{j m}^{\beta}\right|^{p}\right)^{q / p}\right)^{1 / q}
$$

и

$$
\left\|\lambda\left|f_{p q}^{s, \varrho}(M)\left\|=\sup _{\beta \in \mathbb{N}_{0}^{n}} 2^{\varrho|\beta|}\right\|\left(\sum_{j=0}^{\infty} \sum_{m}^{M, j} 2^{j s q}\left|\lambda_{j m}^{\beta}\right|^{q} \chi_{j m}(\cdot)\right)^{1 / q}\right| L_{p}\left(\mathbb{R}^{n}\right)\right\|
$$

с обьчньми изменениями при $p=\infty$ и/или $q=\infty$.

ЗАмечАние 26. Определение 25 - вариант определения 4. Обозначим соответствующие пространства последовательностей через $b_{p q}^{s, \varrho}(M)$ и $f_{p q}^{s, \varrho}(M)$. Возникают некоторые вопросы, особенно в связи с (57). Заметим сначала, что

$$
b_{p p}^{s, \varrho}(M)=f_{p p}^{s, \varrho}(M), \quad 0<p \leqslant \infty .
$$

Можно спросить, что случится, если заменить пространство $L_{p}\left(\mathbb{R}^{n}\right)$ пространством $L_{p}(\Gamma, \mu)$, квазинормированным по формуле

$$
\left\|f \mid L_{p}(\Gamma, \mu)\right\|=\left(\int_{\Gamma}|f(\gamma)|^{p} \mu(d \gamma)\right)^{1 / p}
$$

если $M=\Gamma$ при условии (53). В случае, когда $M=\Omega$, мы рассматриваем (58) для $L_{p}(\Omega)$ с лебеговой мерой $\mu(d x)=d x$ вместо $\mu$. Напомним, что радонова мера $\mu$ 
на $\mathbb{R}^{n}$, удовлетворяющая условию (53), назьвается изотропной, если существует такая непрерывная строго возрастающая функция $h$ на промежутке $[0,1]$, что $h(0)=0$, $h(1)=1$ и

$$
\mu(B(\gamma, r)) \sim h(r) \text { при } \gamma \in \Gamma \text { и } 0<r<1 \text {, }
$$

где $B(\gamma, r)$-шар с центром в $\gamma$ и радиусом $r$. Тогда Г назьвается $h$-множеством. Частньй случай $h(r)=r^{d}$ с $0 \leqslant d \leqslant n$ приводит к $d$-множествам, и $\mu=\mathscr{H}^{d} \mid \Gamma-$ ограничение хаусдорфовой меры $\mathscr{H}^{d}$ в $\mathbb{R}^{n}$ на $\Gamma$. Детальное изучение $h$-множеств можно найти в [4] и [5]. Пусть $f_{p q}^{s, \varrho, \mu}$ - пространства, определенные формулой $(57)$ с $L_{p}(\Gamma, \mu)$ вместо $L_{p}\left(\mathbb{R}^{n}\right)$. Пусть $\Gamma$ есть $d$-множество с $0<d<n$. Тогда

$$
f_{p p}^{s, \varrho, \mu}=f_{p p}^{\sigma, \varrho}(\Gamma)=b_{p p}^{\sigma, \varrho}(\Gamma), \quad 0<p<\infty, \quad s=\sigma-\frac{n}{p}+\frac{d}{p}
$$

Наметим доказательство этого утверждения. Из (43) и (44) следует, что характеристические функции $\chi_{j m}$ кубов $Q_{j m}, Q_{j m} \cap \Gamma \neq \varnothing$, в формуле (57) можно заменить характеристическими функциями шаров с центрами в точках множества Г и с радиусами $\sim 2^{-j}$, покрьваюшими Г и с попарньми расстояниями $\succeq 2^{-j}$. Тогда формула (60) следует из $(59)$ при $h(r)=r^{d}$. Если $p \neq q$, то пространства $f_{p q}^{s, \varrho}(\Gamma)$ и $f_{p q}^{s, \varrho, \mu}$ могут быть не связаны между собой. Тем не менее, в случае изотропных мер $\mu$ пространства $f_{p q}^{s, \varrho, \mu}$, определяемые внутренним образом, могут оказаться лучшим выбором, когда речь идет об определении соответствующих $\mathfrak{F}$-пространств. Может также сушествовать и соответствующий аналог векторнозначного максимального неравенства (43), указьваюший путь к соответствующей теории для $\mathfrak{F}$-пространств, параллельной приведенньм вьше рассмотрениям в $\mathbb{R}^{n}$. В случае $d$-множеств такое максимальное неравенство было использовано в [16; с. 80-81]. Если $\mu$ неизотропна и не имеет свойства удвоения, то пространства $f_{p q}^{s, \varrho}(\Gamma)$ и $f_{p q}^{s, \varrho, \mu}$ (и даже пространства $b_{p q}^{s, \varrho}(\Gamma)$ и (подходяшим образом определенное) $\left.b_{p q}^{s, \varrho}, \mu\right)$ выглядят не связанными между собой. Мы придерживаемся здесь пространств из определения 25 , добавляя некоторые утверждения, связанные с геометрией множества $M$.

ОПРЕДЕЛЕНИЕ 27. (i) Область $\Omega$ в $\mathbb{R}^{n}$ называется областью с регулярной внутренностью, если сушествует такое положительное число $c$, что $|\Omega \cap B| \geqslant c|B|$ для любого шара с центром в $\partial \Omega$ и радиусом, меньшим 1.

(ii) Компактное множество $\Gamma$ в $\mathbb{R}^{n}$ называется пористым , если сушествует такое $\eta$, $0<\eta<1$, что для любого шара $B(x, r)$ с центром в $x \in \mathbb{R}^{n}$ и с радиусом $0<r<1$ сушествует шар $B(y, \eta r)$, удовлетворяюший условиям

$$
B(y, \eta r) \subset B(x, r) \quad \text { и } B(y, r \eta) \cap \Gamma=\varnothing .
$$

ЗАмечАнИЕ 28. Регулярность внутренности области играет роль в связи с атомическим разложением функциональных пространств в негладких областях, см. [10; с. 59]. Пористость возникает из фрактальной геометрии, см. [21; с. 156-158]. Мы использовали это свойство, называемое также условием шаров, в [30; с. 138-141] в связи с функциональными пространствами на фракталах. Там найдена характеризация того, что данное $h$-множество $\Gamma$ - пористое. В частности, любое $d$-множество с $d<n-$ пористое. Кроме того, если $\Gamma$-пористое, то $|\Gamma|=0$. 
ПРЕДЛОЖЕНИЕ 29. (i) Пусть $\Omega$ - область с регулярной внутренностью в $\mathbb{R}^{n}$, и пусть $\widetilde{\chi}_{j m}^{\Omega}$ - характеристическая функиия пересечения $\widetilde{Q}_{j m} \cap \Omega$, где $\widetilde{Q}_{j m}-\kappa у б$ с иентром в $2^{-j}$ т и слиной стороны $2^{-j+2}$. Пусть $p<\infty$. Тогда правая часть формуль (57) с $\widetilde{\chi}_{j m}^{\Omega}$ вместо $\chi_{j m}$ әквивалентна квазинорме в (57) (внутренняя характеризачия).

(ii) Пусть $p<\infty u \Gamma-$ компактное пористое множество в $\mathbb{R}^{n}$. Тогда $f_{p q}^{s, \varrho}(\Gamma)$ не зависит от $q$ и, в частности,

$$
f_{p q}^{s, \varrho}(\Gamma)=b_{p p}^{s, \varrho}(\Gamma) .
$$

ДокАЗАТЕльство. Часть (i) следует из (43) и (44). Что касается части (ii), заметим сначала, что кубы $Q_{j m}$ можно заменить, снова в силу (43) и (44), шарами радиуса $2^{-j}$ с центрами в $Г$ на попарных расстояниях $\succeq 2^{-j}$. Для любого такого шара можно выбрать подшар в соответствии с (61), радиуса $\eta 2^{-j}$, причем можно дополнительно предположить, что расстояние от каждого подшара до Г не меньше $c 2^{-j}$ при некотором $c>0$. Тогда все эти подшары имеют управляемые пересечения, и можно даже предполагать, что все эти подшары имеют попарно непересекающиеся носители. Это доказывает (62).

ЗАмЕчАнИЕ 30 . В частности, если $\Omega$ имеет регулярную внутренность, то функции $\chi_{j m}$ в $(57)$ можно заменить на $\widetilde{\chi}_{j m}$ (на характеристические функции кубов $\widetilde{Q}_{j m}$ ) и пространство $L_{p}\left(\mathbb{R}^{n}\right)$ на $L_{p}(\Omega)$ (квазинормы эквивалентны).

5.2. Функциональные пространства. Здесь множество $M$ снова является либо областью $\Omega$ в $\mathbb{R}^{n}$, либо компактным множеством $\Gamma$ - носителем меры $\mu$, удовлетворяющей условиям (53)-(55). Для унификации обозначений положим $L_{p}(\Omega)=L_{p}(M, \mu)$, если $M=\Omega$ - область. Если область $\Omega$ неограничена, то символом $L_{\infty}\left(\Omega, w_{\sigma}\right)$ обозначим семейство всех $f$ с $w_{\sigma} f \in L_{\infty}(\Omega)$, где $w_{\sigma}(x)=\left(1+|x|^{2}\right)^{\sigma / 2}$. Пусть $k_{j m}^{\beta}$ - те же функции, что и в определении 14 . Кроме того, $b_{p q}^{s, \varrho}(M)$ и $f_{p q}^{s, \varrho}(M)$ имеют тот же смысл, что и в определении 25 и замечании 26.

ОПРЕДЕЛЕНИЕ 31 . Пусть $0<p \leqslant \infty$ ( $p<\infty$ для $\mathfrak{F}$-пространств $), 0<q \leqslant \infty$ и $\varrho \geqslant 0$. Пусть либо

$$
M=\Omega \text {-область в } \mathbb{R}^{n} \text { и } s>0
$$

либо

$$
M=\Gamma \text { с } \mu \in \mathscr{C}^{-\sigma}\left(\mathbb{R}^{n}\right), \quad 0<\sigma \leqslant n, \quad s>\sigma / p,
$$

в соответствии с (53)-(55). Тогда $\mathfrak{B}_{p q}^{s}(M, \mu)$ - множество всех функций $f \in L_{p}(M, \mu)$, которые могут быть представлены в виде

$$
f=\sum_{\beta, j} \sum_{m}^{M, j} \lambda_{j m}^{\beta} k_{j m}^{\beta}, \quad \lambda \in b_{p q}^{s, \varrho}(M),
$$

где ряд абсолютно сходится в $L_{p}(M, \mu)\left(\right.$ с заменой на $L_{\infty}\left(\Omega, w_{\varkappa}\right), \varkappa<0$, если $p=\infty$ и $\Omega$ - неограниченная область). Пусть

$$
\left\|f\left|\mathfrak{B}_{p q}^{s}(M, \mu)\|=\inf \| \lambda\right| b_{p q}^{s, \varrho}(M)\right\|
$$

где нижняя грань берется по множеству всех допустимых представлений (64). Аналогично, $\mathfrak{F}_{p q}^{s}(M, \mu)$ - семейство всех функций $f \in L_{p}(M, \mu)$, допускаюших представление в виде

$$
f=\sum_{\beta, j} \sum_{m}^{M, j} \lambda_{j m}^{\beta} k_{j m}^{\beta}, \quad \lambda \in f_{p q}^{s, \varrho}(M),
$$


где ряд абсолютно сходится в $L_{p}(M, \mu)$. Пусть

$$
\left\|f\left|\mathfrak{F}_{p q}^{s}(M, \mu)\|=\inf \| \lambda\right| f_{p q}^{s, \varrho}(M)\right\|,
$$

где нижняя грань берется по множеству всех допустимых представлений (65).

ЗАмЕчАниЕ 32. Это - непосредственный вариант определения 14, включая комментарий в замечании 15 . Очевидно, в случае областей $M=\Omega$ предпочтительнее писать $\mathfrak{B}_{p q}^{s}(\Omega)$ и $\mathfrak{F}_{p q}^{s}(\Omega)$.

ТеОрема 33. (i) Приведенные выше пространства $\mathfrak{B}_{p q}^{s}(M, \mu)$ и $\mathfrak{F}_{p q}^{s}(M, \mu) \kappa в а-$

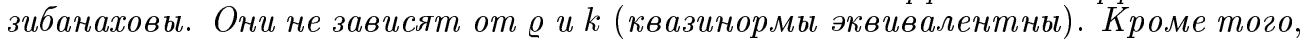
для всех допустимых $s, p$ и q имеем

$$
\mathfrak{B}_{p q}^{s}(M, \mu) \hookrightarrow L_{p}(M, \mu), \quad \mathfrak{F}_{p q}^{s}(M, \mu) \hookrightarrow L_{p}(M, \mu),
$$

$$
\mathfrak{B}_{p, \min (p, q)}^{s}(M, \mu) \hookrightarrow \mathfrak{F}_{p q}^{s}(M, \mu) \hookrightarrow \mathfrak{B}_{p, \max (p, q)}^{s}(M, \mu) .
$$

(ii) Пусть, кроме того, Г в (53) - пористое в смысле определения 27 (ii). Тогда $\mathfrak{F}_{p q}^{s}(\Gamma, \mu)$ не зависит от $q$. В частности,

$$
\mathfrak{F}_{p q}^{s}(\Gamma, \mu)=\mathfrak{B}_{p p}^{s}(\Gamma, \mu)
$$

ДокАЗАТЕльство. Докажем первое включение в (66) для случая множества $M=\Gamma$, удовлетворяюшего условиям (53)-(55). Пусть $p<\infty$ (при $p=\infty$ нужны соответствуюшие изменения). По аналогии с (37) получаем

$$
\begin{aligned}
\left\|\sum_{m}^{\Gamma, j} \lambda_{j m}^{\beta} k_{j m}^{\beta} \mid L_{p}(\Gamma, \mu)\right\|^{p} & \leqslant c 2^{-\varepsilon|\beta| p} \sum_{m}^{\Gamma, j}\left|\lambda_{j m}^{\beta}\right|^{p} \mu_{j} \\
& \leqslant c^{\prime} 2^{-\varepsilon|\beta| p} 2^{-(n-\sigma) j} 2^{-j s p+j n} \leqslant c^{\prime} 2^{-\varepsilon|\beta|} 2^{-j(s p-\sigma)} .
\end{aligned}
$$

Из (63) следует первое включение в (66). Все остальные утверждения в части (i) можно получить так же, как в доказательстве теоремы 16. Часть (ii) следует из предложения 29 (ii).

5.3. Свойства, комментарии, преобразования типа “снежинки". Здесь собраны некоторые замечания и ссылки на близкие по тематике работы.

ЗАмечАнИЕ 34 . Пусть $M=\Omega$ - область в $\mathbb{R}^{n}$. Пусть $0<p \leqslant \infty(p<\infty$ для $\mathfrak{F}$-пространств), $0<q \leqslant \infty$ и $s>0$. Тогда из определений 14 и 31 следует, что пространства на $\Omega$ являются ограничениями соответствуюших пространств на $\mathbb{R}^{n}$ в обычном смысле,

$$
\left\|f\left|\mathfrak{B}_{p q}^{s}(\Omega)\|=\inf \| g\right| \mathfrak{B}_{p q}^{s}\left(\mathbb{R}^{n}\right)\right\|, \quad g \mid \Omega=f,
$$

как подпространства в $L_{p}(\Omega)$; ситуация для $\mathfrak{F}$-пространств аналогична. Так как пространства анализа Фурье, $B_{p q}^{s}(\Omega)$ и $F_{p q}^{s}(\Omega)$, также определяются ограничениями соответствуюших пространств на $\mathbb{R}^{n}$, то, в частности, получаем равенства

$$
\mathfrak{B}_{p q}^{s}(\Omega)=B_{p q}^{s}(\Omega) \text { при } s>\sigma_{p}, \quad \mathfrak{F}_{p q}^{s}(\Omega)=F_{p q}^{s}(\Omega) \text { при } s>\sigma_{p q},
$$

как следствие теоремы 18. 
ЗАмЕчАнИЕ 35. Пусть $\mu$ и Г - как в (53)-(55). В [29], [30] и недавно в [35; $11.17 .2,7]$, мы имели дело с пространствами на $\Gamma=\operatorname{supp} \mu$. В частности, при $0<1 / p=t<1$, $0<q \leqslant \infty$ и $s>0$ мы очень естественно ввели пространства

$$
B_{p q}^{s}(\Gamma, \mu)=\operatorname{tr}_{\mu} B_{p q}^{s+\left|s_{\mu}(1-t)\right|}\left(\mathbb{R}^{n}\right)
$$

как пространства следов, где $s_{\mu}(1-t)$ - характеристика Бесова радоновой меры $\mu$. Но мы здесь не обсуждаем детали. Заметим только, что в случае $d$-множеств, которые мы кратко упомянули после (59), имеем $\left|s_{\mu}(1-t)\right|=t(n-d)$. Если $s+\left|s_{\mu}(1-t)\right|>\sigma t$ с теми же $\mu$ и $\sigma$, что и в (63), то $s>0$ и

$$
B_{p q}^{s}(\Gamma, \mu)=\mathfrak{B}_{p q}^{s+\left|s_{\mu}(1-t)\right|}(\Gamma, \mu) .
$$

Подход через следы (пока что) тоньше при $1<p<\infty$. С другой стороны, $\mathfrak{B}$-пространства (в виде, введенном в определении 31 ) применимы ко всем $0<p \leqslant \infty$.

ЗАМЕчАнИЕ 36 . В литературе есть несколько предложений введения функциональных пространств на абстрактных пространствах $(X, \varrho, \mu)$, где $X$ - множество, $\varrho$ - (квази-)метрика и $\mu$ - соответствуюшая борелевская мера. В случае соболевских пространств (первого порядка) сошлемся на [14]. Другой подход к пространствам типа Бесова на компактных множествах $\Gamma$ в $\mathbb{R}^{n}$ основан на наблюдении, что $\Gamma$ можно снабдить мерой со свойством удвоения, [36], и кратко упомянут в начале $\S 5.1$. Уточнения этого подхода и соответствуюших пространств Бесова, основанные на (первых) разностях и атомах, приведены в [20] и [6]. Кроме того, более подробные ссылки можно найти в $[35 ; \S 1.17 .5]$. Ближе к нашей теме находятся пространства типа $B_{p q}^{s}(X)$ и $F_{p q}^{s}(X)$ на пространствах $(X, \varrho, \mu)$ однородного типа, особенно при ограничении на случай $1 \leqslant p \leqslant \infty$ (некоторые расширения на случай $p<1$ тоже есть), $1 \leqslant q \leqslant \infty$ и $|s|<1$, где $\mu$ - борелевская мера со свойством удвоения. Подробные ссылки см. в $[35 ; \S 1.17 .5]$. Отметим здесь обзоры $[15]$ и $[16]$. В случае пространств $F_{p q}^{s}(X)$ соответствующая техника может оказаться ближе к технике пространств последовательностей $f_{p q}^{s, \varrho, \mu}$, кратко упомянутой между (59) и (60), чем к технике пространств типа $f_{p q}^{s, \varrho}$, примененной выше в надежде, что найдется вариант мошного инструмента векторнозначных максимальных неравенств вида (43), что было использовано в [16]. Но, по-видимому, это требует некоторой регулярности меры $\mu$, например, изотропности. Соответствующая часть утверждения (43) недостижима для общих мер. Тем не менее есть способ обойти эту трудность, см. [17]. Следует заметить, что теория пространств $B_{p q}^{s}(X)$ и $F_{p q}^{s}(X)$ - в том виде, в котором она развита в [15]-[17] (с векторнозначными максимальными неравенствами Харди-Литлвуда или без них) - с одной стороны и подход, представленньй в настояшей работе, с другой, имеют нечто общее. Оба подхода решительно зависят от воспроизводяших формул типа (5) и их абстрактных вариантов в $(X, \varrho, \mu)$.

ЗАмечАнИЕ 37 . Введенное выше однородное пространство $(X, \varrho, \mu)$ назьвается $d$-пространством при некотором $d>0$, если мера $\mu$, удовлетворяющая условию удвоения, удовлетворяет и условию

$$
\mu(B(x, r)) \sim r^{d} \text { при } x \in X \text { и } 0<r \leqslant \operatorname{diam} X<\infty,
$$

где $B(x, r)$ - шар радиуса $r$ с центром в $x$. Сушествует такое число $0<\varepsilon_{0} \leqslant 1$, что для любого $\varepsilon$ с $0<\varepsilon \leqslant \varepsilon_{0}$ можно найти билипшицево отображение $H \mapsto \mathbb{R}^{n}$,

$$
H:\left(X, \varrho^{\varepsilon}, \mu\right) \Longleftrightarrow\left(\Gamma, \varrho_{n}, \mathscr{H}^{d / \varepsilon} \mid \Gamma\right),
$$


так назьваемой версии типа "снежинки" $\left(X, \varrho^{\varepsilon}, \mu\right)$ пространства $(X, \varrho, \mu)$ на заданное $d / \varepsilon$-множество $\Gamma$ в некотором (многомерном) пространстве $\mathbb{R}^{n}$ с $d / \varepsilon<n$, где $\varrho_{n}$ - соответствуюшая евклидова метрика и $\mathscr{H}^{d / \varepsilon}-$ связанная с ней хаусдорфова мера. Это - так назьваемое преобразование типа “снежинки” пространства $(X, \varrho, \mu)$. Оно дает возможность перенести пространство

$$
B_{p q}^{s}(\Gamma, \mu)=\mathfrak{B}_{p q}^{s+\frac{n-d / \varepsilon}{p}}(\Gamma, \mu)
$$

(отвечающее формуле $(67))$ в $d$-пространство $(X, \varrho, \mu)$, и поэтому получить формулу

$$
f=\sum_{\beta, j} \sum_{m}^{M, j} \lambda_{j m}^{\beta} H^{-1} k_{j m}^{\beta}
$$

в качестве перенесенного представления (65). В частности, $H^{-1} k_{j m}^{\beta} \geqslant 0$. Это подробно сделано в [34] в предположении, что $s>0$ и $1<p=q<\infty$. Теперь эта конструкшия может быть распространена на все родственные пространства, рассматриваемые в определении 31 и теореме 33. Преобразование типа "снежинки" восходит к [2]. Мы также отсылаем читателя к подробному тексту [19]. Описание и дальнейшие ссылки можно также найти в [34] и [35; 11.17 .9$]$. Но основным наблюдением в связи с нашим подходом является то, что это преобразование типа "снежинки" работает в гораздо более богатой шкале квазиметрических пространств $(X, \varrho, \mu)$ с мерами, имеющими свойство удвоения [19]. Более подробные ссылки даны в [34] и [35; §1.17]. На основании определения 31 этим способом можно перенести $\mathfrak{B}^{s}$-пространства на широкие классы однородных пространств $(X, \varrho, \mu)$ для всех $s>0,0<p \leqslant \infty$ и $0<q \leqslant \infty$. Но это еще не сделано.

\section{СПИСОК ЛИТЕРАТУРЫ}

[1] D. R. Adams, L. I. Hedberg. Function Spaces and Potential Theory. Berlin: SpringerVerlag, 1996.

[2] P. Assouad. Plongements lipschitziens dans $\mathbb{R}^{n} / /$ Bull. Soc. Math. France. 1983. V. 111. № 4. P. 429-448.

[3] О.В. Бесов, В.П. Ильин, С. М. Никольский. Интегральные представления функций и теоремы вложения. М.: Наука, 1975.

[4] M. Bricchi. Complements and results on $h$-sets // Function Spaces, Differential Operators and Nonlinear Analysis. Basel: Birkhäuser, 2003. P. 219-229.

[5] M. Bricchi. Tailored Besov spaces and $h$-sets // Math. Nachr. 2004. V. 263/264. P. 36-52.

[6] P. Bylund. Besov spaces and measures on arbitrary sets // Ph.D Thesis. Umeå: University of Umeå, 1994.

[7] M. Christ, A. Seeger. Necessary conditions for vector-valued operator inequalities in harmonic analysis // ArXiv: math.CA/0504030.

[8] R.A. DeVore, G.G. Lorentz. Constructive Approximation. Berlin: Springer-Verlag, 1993.

[9] R. A. DeVore, R. C. Sharpley. Besov spaces on domains in $\mathbb{R}^{d} / /$ Trans. Amer. Math. Soc. 1993. V. 335. № 2. P. 843-864.

[10] D. E. Edmunds, H. Triebel. Function Spaces, Entropy Numbers, Differential Operators. Cambridge: Cambridge Univ. Press, 1996.

[11] C. Fefferman, E. M. Stein. Some maximal inequalities // Amer. J. Math. 1971. V. 93. P. $107-115$.

[12] M. Frazier, B. Jawerth. A discrete transform and decompositions of distribution spaces // J. Funct. Anal. 1990. V. 93. № 1. P. 34-170.

[13] M. Frazier, B. Jawerth, G. Weiss. Littlewood-Paley Theory and the Study of Function Spaces. Providence, RI: Amer. Math. Soc., 1991. 
[14] P. Hajłasz, P. Koskela. Sobolev met Poincaré // Mem. Amer. Math. Soc. 2000. V. 145. № 688. P. 1-101.

[15] Y.S. Han, E. T. Sawyer. Littlewood-Paley theory on spaces of homogeneous type and classical function spaces // Mem. Amer. Math. Soc. 1994. V. 110. № 530.

[16] Y. Han, D. Yang. New characterizations and applications of inhomogeneous Besov and Triebel-Lizorkin spaces on homogeneous type spaces and fractals // Dissertationes Math. (Rozprawy Mat.). 2002. V. 403.

[17] Y. Han, D. Yang. Triebel-Lizorkin spaces with non-doubling measures // Studia Math. 2004. V. 162. № 2. P. 105-140.

[18] L. I. Hedberg, Yu. Netrusov. An axiomatic approach to function spaces, spectral synthesis, and Luzin approximation // Mem. Amer. Math. Soc. (to appear).

[19] J. Heinonen. Lectures on Analysis on Metric Spaces. New York: Springer-Verlag, 2001.

[20] A. Jonsson. Besov spaces on closed subsets of $\mathbb{R}^{n} / /$ Trans. Amer. Math. Soc. 1994. V. 341. № 1. P. $355-370$.

[21] P. Mattila. Geometry of Sets and Measures in Euclidean Spaces. Cambridge: Cambridge Univ. Press, 1995.

[22] Ю. Нетрусов. Множества особенностей функций из пространств типа Бесова и Лизоркина-Трибеля // Труды МИАН. 1989. Т. 187. С. 162-177.

[23] С. М. Никольский. Приближение функций многих переменных и теоремы вложения. М.: Наука, 1969.

[24] С. Л. Соболев. Некоторые применения функционального анализа в математической физике. Л.: Изд-во Ленингр. ун-та, 1950.

[25] Э. А. Стороженко, П. Освальд. Теорема Джексона в пространствах $L^{p}\left(R^{k}\right), 0<$ $p<1$ // Сиб. матем. журн. 1978. Т. 19. № 4. С. 888-901.

[26] A. Torchinsky. Real-variable Methods in Harmonic Analysis. Orlando, FL: Academic Press, 1986

[27] Н. Triebel. Theory of Function Spaces. Basel: Birkhäuser, 1983. (Рус. пер.: Х. Трибель. Теория функциональных пространств. М.: Мир, 1986.)

[28] H. Triebel. Theory of Function Spaces. II. Basel: Birkhäuser, 1992.

[29] H. Triebel. Fractals and Spectra. Related to Fourier Analysis and Function Spaces. Basel: Birkhäuser, 1997.

[30] H. Triebel. The Structure of Functions. Basel: Birkhäuser, 2001.

[31] H. Triebel. Wavelet frames for distributions; local and pointwise regularity // Studia Math. 2003. V. 154. № 1. P. 59-88.

[32] H. Triebel. Fractal characteristics of measures: An approach via function spaces // J. Fourier Anal. Appl. 2003. V. 9. № 4. P. 411-430.

[33] H. Triebel. The positivity property of function spaces // Proceedings of the Sixth Conference "Function Spaces" (Wroclaw, 2001) / ed. R. Grzaslewicz et al. River Edge, NJ: World Scientific, 2003. P. 263-274.

[34] H. Triebel. A new approach to function spaces on quasi-metric spaces // Rev. Mat. Complut. 2005. V. 18. № 1. P. 7-48.

[35] H. Triebel. Theory of Function Spaces. III. Basel: Birkhäuser (to appear).

[36] А. Л. Вольберг, С. В. Конягин. О мерах с условием удвоения // Изв. АН СССР. Сер. матем. 1987. Т. 51. № 3. С. 666-675.

[37] P. Wojtaszczyk. A Mathematical Introduction to Wavelets. Cambridge: Cambridge Univ. Press, 1997.

Mathematisches Institut,

Поступила в редакцию

Friedrich-Schiller-Universität, Jena, Germany

20.09 .2005

E-mail: triebel@minet.uni-jena.de 